\title{
A Rare Case of Primary Pulmonary Amoebiasis without Gastrointestinal Involvement: A Case Report
}

\author{
Arie Gradiyanto Nugroho ${ }^{1 *}$ (ID) , Edijono $^{\hat{\imath}}$ \\ ${ }^{1}$ Emergency Department, Dr. Ramelan Naval Hospital, Surabaya, Indonesia. \\ ${ }^{2}$ Department of Pulmonology and Respiratory Medicine, Dr. Ramelan Naval Hospital, Surabaya, Indonesia.
}

\section{ARTICLE INFO}

Article history:

Received 17 November 2020

Received in revised form 28

September 2021

Accepted 29 September 2021

Available online 30 September 2021

\section{Keywords:}

Amoebiasis,

Infectious disease,

Lung abscess,

Pulmonary amoebiasis.

\begin{abstract}
Introduction: The main pathogen of amoebiasis is Entamoeba histolytica which is very common in tropical and developing countries, where sanitation, hygiene, and low socioeconomic status are major problems. The most common site of infection is the intestinal mucosa. For extraintestinal amoebiasis, the most common sites are the liver, followed by the lungs and brain.

Case: A 15-year-old male was presented to the ER with chief complaint of breathlessness for the last 10 days, accompanied by productive coughing with dark brown sputum and pain on lower right chest. The patient had fever while the disease progressed. Gastrointestinal symptoms were denied. Physical examination showed lowered breathing sound on the right lung, dullness on lower right chest, and usage of accessory breathing muscles. USG found heteroechoic lesion of $8 \times 7 \mathrm{~cm}$ in size suspicious of lung abscess, later on confirmed as Entamoeba histolytica found in sputum sample, while abdominal USG showed no abnormality. The patient was admitted for antibiotic therapy and consultation to cardiothoracic surgeon.

Discussion: One of the rarest routes of amoebiasis is primary deposition of cysts to the lungs through aspiration. Pulmonary amoebiasis often causes abscess formation, produces brown-colored sputum called "anchovy sauce". It is notable that we found one of the rarest cases of amoebiasis infection, where there is a pulmonary infection without any intestinal involvement.

Conclusion: Whilst infection of primary pulmonary amoebiasis is very rare, it is still an important etiology to put on the differential diagnosis of pulmonary abscess. Therefore, sputum examination or biopsy is required even when there are no gastrointestinal disturbances.
\end{abstract}

\section{INTRODUCTION}

Amoebiasis is one of the most common parasitic disease caused by Entamoeba infection. The main pathogen of amoebiasis is Entamoeba histolytica. Amoebiasis is very common in tropical and developing countries, such as Mexico, Central and South America, Africa, and the Indian Subcontinent, where sanitation, hygiene, and low socio-economic status are major problems. Nowadays, supported by easier travel between countries, it becomes a globalized disease. Amoebiasis is currently estimated to cause 50 million cases a year with 40,000 to 100,000 deaths worldwide. Most of the amoebic infection found in human gastrointestinal tract are asymptomatic. Up to $90 \%$ of the people infected with amoeba are carriers, which carry nonpathogenic Entamoeba dispar rather than the pathogenic Entamoeba histolytica. ${ }^{1,2}$
Entamoeba histolytica has two life cycles, one as an infectious trophozoite and the second life cycle as a cyst where it can survive in harsh environment outside a host's body weeks to months thanks to its chitinous cell wall. The most common route of infection is through gastrointestinal tract, where a mature cyst is ingested through fecal material which is contaminated in food, water, hands, or sexual contact, followed by excystation, releasing trophozoites into the small intestine. The released trophozoites then multiply in the lumen of the intestine, some may inhabit intestinal lumen causing noninvasive infection, others invade intestinal mucosa or blood vessels to infect extraintestinal organs such as liver, brain, and lungs. Cysts can be formed through binary fission and excreted through feces to infect subsequent hosts. Trophozoites

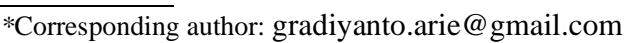


can also be found in feces, although this form is rapidly destroyed in the environment, thus cannot be transmitted to other hosts. ${ }^{1,3}$

Trophozoites adherence to the mucus layer is made possible through the Gal.GalNAc lectin adhesion molecule which targets the $\mathrm{N}$-terminal galactose or $\mathrm{N}$ acetyl-D-galactosamine to adhere to. After adhering to the mucosa, certain enzymes such as sialidase, $\mathrm{N}$ acetylgalactosamidase, and $\mathrm{N}$-acetylglucosaminidase are produced to degrade the mucus barrier, enabling trophozoites to penetrate mucosal wall to invade extraintestinal organs and blood vessels. These specific process causes certain hosts lacking the galactose molecule virtually harder to invade, and those amoeba species which do not possess the enzymes required unable to cause invasive disease. Degradation of mucus barrier, cytolysis, and apoptosis releases interleukin- $1 \alpha$ and interleukin- $1 \beta$ which activates NF-kB in distal cells to produce inflammatory mediators. The release of inflammatory mediators attracts neutrophils and macrophages which then is destroyed by trophozoites resulting in more mediators release and increases inflammation further. ${ }^{4,5}$

Entamoeba histolytica most commonly affects gastrointestinal tract, where typically causes subacute onset of diarrhea, bloody stool, weight loss, and abdominal pain. Rarely, nectrotizing colitis, toxic megacolon, and perianal ulceration can occur, especially when there is delay in diagnosis and treatment. Immunocompromised people such as malnourished people, pregnant women, corticosteroid abusers, and very young children are at an increased risk of developing more severe complications. ${ }^{4-6}$

Extraintestinal amoebiasis varies from the most frequent amoebic liver abscess, pulmonary amoebiasis, cardiac infection, or even brain infection. Most extraintestinal amoebiasis are preceded by amoebic liver abscess and occurs by direct spread from amoebic liver abscess. For example, hepatobronchial fistula or hepatocardiac fistula formation usually occurs from a rupture of a superior right lobe abscess which erodes through the diaphragm and spreads to pleural space or left liver lobe abscess to the pericardium. Although unlikely, it is also possible for them to occur hematogenously without hepatic involvement. ${ }^{4-7}$

Symptoms of an uncomplicated pulmonary amoebiasis is closely related to pneumonia, including fever, shivering, productive cough, fatigue, and dyspnea. The main pathological feature of pulmonary amoebiasis is abscess formation, where bacterial toxin, vasculitis, and proteases cause destruction-forming area of necrotic lung tissue. When lung abscess is formed and connected to the bronchus, it produces brown-colored cough sputum called "anchovy sauce sputum". 1,8

X-ray remains the first line supporting examination, providing fast results which can raise the suspection of pulmonary amoebiasis when lung abscess is found. Amoebiasis frequently has iregullar thickwalled cavity with or without air fluid level with nearby infiltrates. USG and CT scan can greatly support the diagnosis of lung abscess and discover the existence of hepatobiliary abscess. ${ }^{8,9}$

Early sample collection for laboratory needs to be prioritized in order to confirm the diagnosis and main etiological culprit of said infection. Late sample collection can lead to late diagnosis which increases the risk of the disease evolving to more severe condition. Key laboratory finding is trophozoites presence in sputum or stool sample, obtained through expectoration or excretion, or from invasive procedures such as transtracheal aspiration, transthoracic aspiration, and fiber optic bronchoscopy, depending on the site of infection. Entamoeba histolytica and Entamoeba dispar are indistinguishable by microscopic examination, requiring $\mathrm{PCR}$ and serology tests which are highly specific and sensitive to detect parasitic DNA, although PCR is much more costly compared to morphological sputum or stool examination. ${ }^{1,7-9}$

Management of pulmonary amoebiasis includes antibiotic regimen, abscess drainage, chest physiotherapy, and surgery for advanced infection. Current treatment of choice is antibiotic regime of ten days of $750 \mathrm{mg}$ Metronidazole IV treatment three times a day while monitoring clinical response and radiographic appearance. Tinidazole is an alternative to Metronidazole with similar efficacy and toxicity. Following either Methronidazole or Tinidazole treatment, it is recommended to administer Paromomycin (25-30 mg/kg/day orally in three divided doses for seven days) and diloxanide furoate $(500 \mathrm{mg}$ orally three times daily for 10 days) to eradicate cysts in the intestinal lumen, prevcenting furter transmission or relapse. While most liver abscesses can be treated without percutaneous aspiration, large $(>300 \mathrm{~mL})$ pleural or paracardial effusion should undergone drainage to improve recovery rate. Passive chest physiotherapy such as postural drainage helps to remove sputum and prevent sputum retention. In cases with very large abscesses, resistant organism, hemorrhage, or recurrent disease, thoracotomy or lobectomy is also indicated. ${ }^{1,8,10}$ 
Although amoebiasis most commonly affects gastrointestinal tract, and while it is possible to cause extraintestinal amoebiasis, most of them are preceded by amoebic liver abscess. In this case, we present a rare case of a primary pulmonary amoebiasis without gastrointestinal involvement.

\section{CASE}

A 15-year-old male was presented to the ER with chief complaint of breathlessness for the last 10 days which was not affected by activity. He also complained of productive coughing with dark brown sputum (Figure 1) and chest pain on the lower right chest. The patient acknowledged experiencing fever while the disease progressed. Nausea, abdominal pain, diarrhea, bloody stool, or other gastrointestinal symptoms were denied.

Initial physical examination showed that the patient was fully aware with GCS score of 15 (4-5-6), blood pressure was $124 / 78 \mathrm{mmHg}$, heart rate was 132 bpm, body temperature of $37.7^{\circ} \mathrm{C}$, respiratory rate was $24 \mathrm{bpm}$, and $\mathrm{SpO}_{2}$ of $97 \%$ room air. His chest was symmetrical, lowered breathing sound on the right lung, dullness on lower right chest upon percussion, and usage of accessory breathing muscles. No other physical abnormalities found.

The patient got oxygen supplementation, undergone chest X-ray, and got his blood checked. Xray examination showed air-fluid level on right lower lobe, indicating a lung abscess with infiltrates (Figure $2 b)$. The results of blood tests at hospital admission showed increased leukocyte count (18.760 cells/uL) dominated by neutrophils (15.700 cells/uL / 83.7\%). Blood gas analysis using 8 liter/minute oxygen via a simple mask (SM) showed $\mathrm{pH} 7.457, \mathrm{pCO}_{2} 34.6 \mathrm{mmHg}$, $\mathrm{pO}_{2} 166.7 \mathrm{mmHg}, \mathrm{HCO}_{3} 23.9 \mathrm{mmol} / \mathrm{L}, \mathrm{BE} 0.0 \mathrm{mEq} / \mathrm{L}$, $\mathrm{SaO}_{2} 99.2 \%$, and the impression of a respiratory alkalosis, while antigen nasopharyngeal swab was negative for SARS-CoV-2. Sputum and stool sample were collected and Entamoeba histolytica was discovered in the sputum sample, while stool sample showed no abnormalities. Further examination of thoracal USG found heteroechoic lesion of $8 \times 7 \mathrm{~cm}$ in size suspicious of lung abscess (Figure 3). Thoracal CT scan indicated abscess in the inferior lobe of right lung accompanied with empyema (Figure 4), while abdominal USG showed no abnormality in liver or other abdominal organs.

The patient was diagnosed with pulmonary amoebiasis and was admitted for antibiotic therapy using combination of $500 \mathrm{mg}$ metronidazol infusion three times a day and $750 \mathrm{mg}$ levofloxacin infusion once a day, peroral paracetamol and codein as needed, and consultation to cardiothoracic surgeon. The patient eventually got better after 10 days of treatment, fever and cough had subsided, and the breathlessness had significantly reduced. After 5 days without fever and 2 days without productive cough, the patient was discharged and put on outpatient clinic visit for scheduled inferior lobectomy. During the preparation of surgical re-examination, chest X-ray showed significant improvement (Figure 5), thus the inferior lobectomy was cancelled.

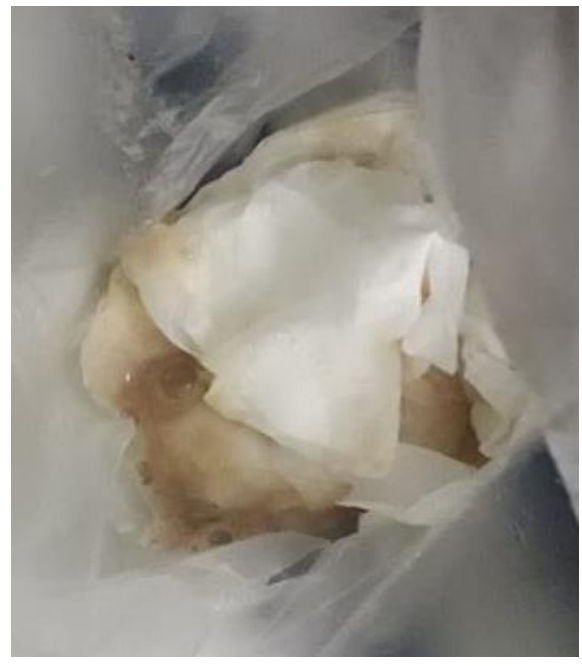

Figure 1. Dark brown sputum produced by the patient which then showed Entamoeba histolytica in microscopic examination.

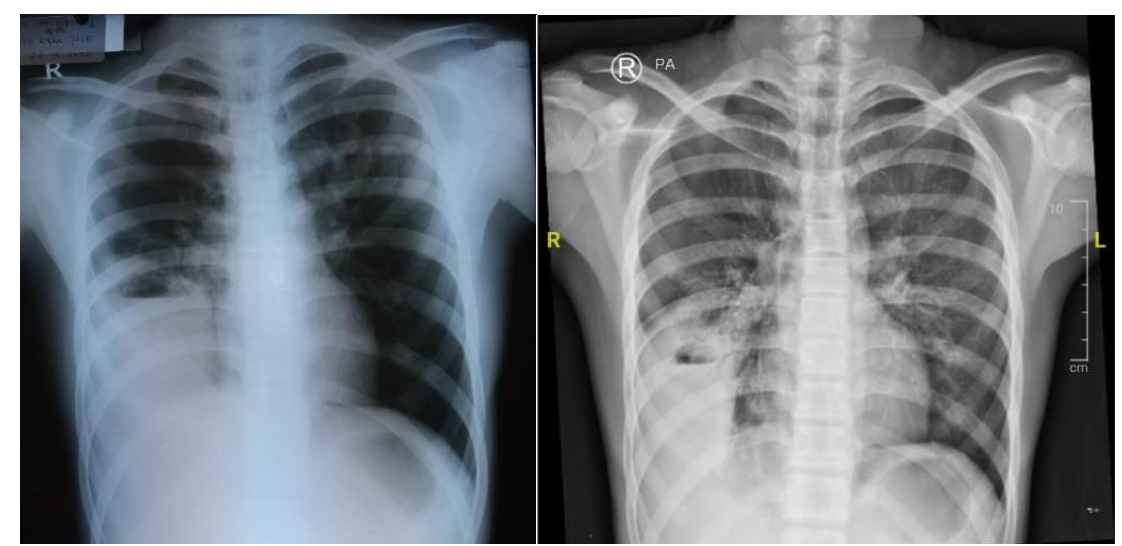

Figure 2. Chest X-ray taken on (a) 28 October 2020, radiological examination performed outside the referral hospital. (b) evaluation of radiological examination on 9 November 2020, performed at the referral hospital, showed homogen opacity with air-fluid level (arrow) 


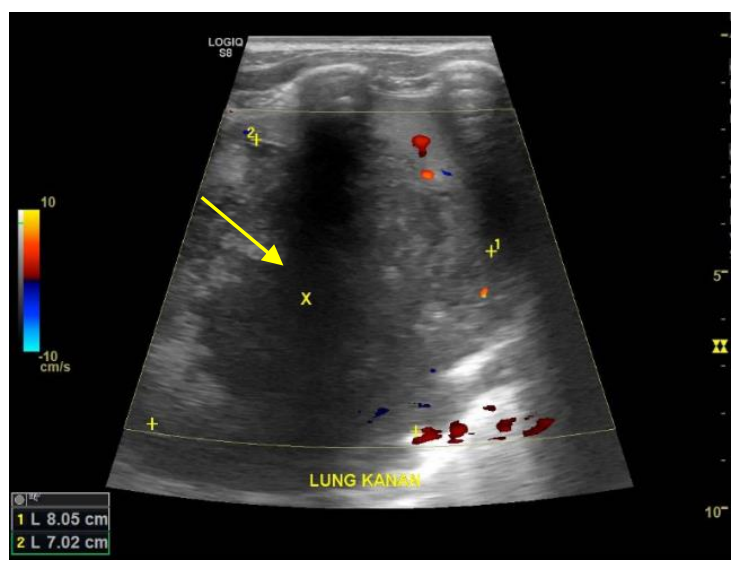

Figure 3. Thoracal USG performed on 2 November 2020 showed heteroechoic lesion of $8 \times 7 \mathrm{~cm}$ in size (arrow) suspicious of lung abscess.

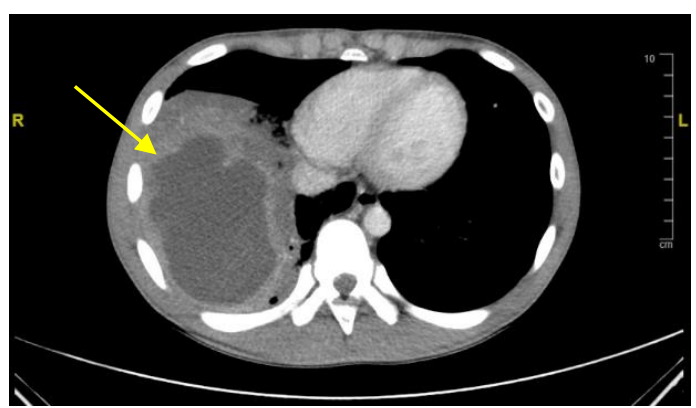

Figure 4. Chest CT-Scan performed on 2 November 2020 showed abscess in the inferior lobe of right lung accompanied with empyema (arrow).

\section{DISCUSSION}

In this case, all sign and symptoms manifested on the patient since first presentation already pointed at lung abscess, from coughing with dark brown sputum as seen in Figure 1, fever, dullness of lower right chest on percussion, lower right chest pain, to dyspnea. Air fluid level in chest X-ray examination also supported the diagnosis of lung abscess (Figure 2), and heteroechoic lesion in chest USG confirmed the existence of abscess (Figure 3). Luckily, the patient could spontaneously produce his dark brown sputum, unique for amoebiasis infection, which confirmed the diagnosis when microscopic examination found Entamoeba histolytica infection, eliminating the need of requiring biopsy or other invasive procedure to acquire abscess sample.

Amoebiasis is caused by Entamoeba infection. The main pathogen of amoebiasis is Entamoeba histolytica. The most common site of infection is the intestinal mucosa causing intestinal disease. For extraintestinal amoebiasis, the most common sites are the liver, followed by the lungs, pericardium, and brain. Usually, pulmonary involvement in amoebiasis occurs when trophozoites from gastrointestinal tract invades the liver, creating liver abscess which then spreads to the

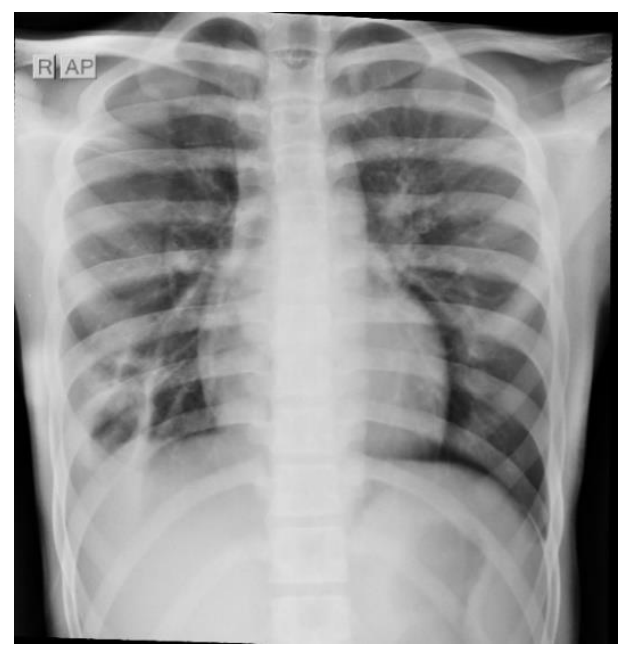

Figure 5. Chest X-ray taken on 22 November 2020 showed significant improvement of the lungs (arrow).

pleura and lungs through diaphragm or via bloodstream. In this case, we found one of the rarest routes of Entamoeba histolytica infection, which is primary deposition of cysts to the lungs through aspiration without any intestinal involvement. This is supported by the fact that there were no disturbances on the gastrointestinal tract. Supporting examination, such as stool examination, found neither cysts nor trophozoites, while abdominal ultrasound also excluded liver abscess, one of the most common finding in amoebiasis infection.

There is only a handful of similar documented cases where primary pulmonary amoebiasis is concerned. Some of them were a 56-year-old $\operatorname{man}^{9}$, a 72-year-old woman ${ }^{11}$, and a 24-year-old man with a history of down syndrome ${ }^{11}$ who was diagnosed with primary pulmonary amoebiasis. In the article published by Daya, R.C., ${ }^{11}$ five out of nine reported pleuropulmonary amoebiasis cases had no hepatobiliary involvement, consisting of 2 adults aged 49 and 56 years old, and 3 elderlies aged 70, 72, and 78 years old. This pattern shows most patients who got primary pulmonary amoebiasis were adults, elderlies, or young man with history of past illnesses which might render them immunodeficient, but this case was a young man without a history of past illnesses.

In Aissa, et al.'s article, the patient was initially treated with antibiotics aimed for community acquired pneumonia which did not work well on the patient, before the parasitological test showed the presence of Entamoeba histolytica. It is concluded that in assessing respiratory diseases and lung abscess suspicion, although lung amoebiasis without gastrointestinal problem is very rare, it is important not to forget Entamoeba histolytica infection as a different diagnosis. Early abscess microscopic examination, either from sputum produced by the patient or by invasive biopsy, if necessary, is crucial to confirm etiology of infection and guide the choice of treatment. ${ }^{9}$ 


\section{CONCLUSION}

Whilst infection of primary pulmonary amoebiasis is very rare, it is still an important etiology to put on the differential diagnosis of pulmonary abscess. Therefore, sputum examination or biopsy is required even when there are no gastrointestinal disturbances.

\section{ACKNOWLEDGMENT}

We would like to thank Edijono, MD. for the contribution in supervising and advising the creation of this article.

\section{REFERENCES}

1. Murray JF, Nadel JA, Broaddus VC, et al. Murray and Nadel's Textbook of Respiratory Medicine. 6th ed. Philadelphia: W.B. Saunders, 2016. Epub ahead of print 2016. DOI: https://doi.org/10.1016/B978-1-4557-33835.00135-4.

2. Roure S, Valerio L, Soldevila L, et al. Approach to Amoebic colitis: Epidemiological, Clinical and Diagnostic Considerations in a Non-Endemic Context (Barcelona, 2007-2017). PLoS One 2019; 14: e0212791.

3. Prevention $\mathrm{C}$ for $\mathrm{DC}$ and. Amebiasis. $D P D x$ Laboratory Identification of Parasites of Public Health Concern https://www.cdc.gov/dpdx/amebiasis/index.html (2019, accessed 27 September 2021).

4. Kantor M, Abrantes A, Estevez A, et al. Entamoeba Histolytica: Updates in Clinical
Manifestation, Pathogenesis, and Vaccine Development. Can J Gastroenterol Hepatol 2018; 2018: 4601420.

5. Chou A, Austin RL. Entamoeba Histolytica. In: StatPearls. Treasure Island (FL): StatPearls Publishing, 2021.

6. Shirley D-A, Hung C-C, Moonah S. Entamoeba histolytica (Amebiasis). In: Ryan ET, Hill DR, Solomon T, et al. (eds) Hunter's Tropical Medicine and Emerging Infectious Diseases. London: Elsevier, 2020, pp. 699-706.

7. Matsuo T, Saito A, Kawai F, et al. Use of PCR in the Diagnosis of Pericardial Amebiasis: A Case Report and Systematic Review of the Literature. BMC Infect Dis 2021; 21: 960.

8. Amin M, Koesoemoprodjo W, Hasan H, et al. Buku Ajar Paru 2019. Surabaya: Departemen/SMF Pulmonologi dan Ilmu Kedokteran Respirasi Fakultas Kedokteran Universitas Airlangga/RSUD Dr. Soetomo, 2019.

9. Aissa A, Hachicha M, Daadoucha A, et al. Pleuropulmonary Amoebiasis: Know to Think About. J Pulm Respir Med; 7. Epub ahead of print 1 January 2017. DOI: 10.4172/2161105X.1000409.

10. Zakaria A, Al-Share B, Al Asad K. Primary Pulmonary Amebiasis Complicated with Multicystic Empyema. Case Rep Pulmonol 2016; 2016: 8709347.

11. Daya RC. A Case of Primary Pulmonary Amoebiasis. In: D49. Lung Infection Case Reports II: Infections other than Fungal Infections. American Thoracic Society, 2016, pp. A7197-A7197. 